Relations industrielles

Industrial Relations

\title{
Plaidoyer pour la grande organisation, Léonard R. Sayles, Entreprise Moderne d'Édition, Paris, 1965, 141 pages.
}

\section{Ronald Pleau}

Volume 20, numéro 4, 1965

URI : https://id.erudit.org/iderudit/027633ar

DOI : https://doi.org/10.7202/027633ar

Aller au sommaire du numéro

Éditeur(s)

Département des relations industrielles de l'Université Laval

ISSN

0034-379X (imprimé)

1703-8138 (numérique)

Découvrir la revue

Citer ce compte rendu

Pleau, R. (1965). Compte rendu de [Plaidoyer pour la grande organisation, Léonard R. Sayles, Entreprise Moderne d’Édition, Paris, 1965, 141 pages.] Relations industrielles / Industrial Relations, 20(4), 720-721.

https://doi.org/10.7202/027633ar

Tous droits réservés (C) Département des relations industrielles de l'Université Laval, 1965
Ce document est protégé par la loi sur le droit d'auteur. L'utilisation des services d'Érudit (y compris la reproduction) est assujettie à sa politique d'utilisation que vous pouvez consulter en ligne.

https://apropos.erudit.org/fr/usagers/politique-dutilisation/ 
Afin de ne pas devenir vite des gens dépassés, les millions de cadres qui existent tont oux Etots-Unis qu'en France sentiront tôt ou tard au cours de leur carrière le besoin de parfaire leur connaissance. $\mathrm{Ce}$ souci de s'adapter continuellement est essentiel à leur avancement.

Le perfectionnement des cadres est également vital pour la vivacité de l'entreprise et de la nation, laquelle est en harmonie uvec. les ressources humaines.

Après nous avoir défini les cadres et nous ovoir entretenu de propos concernant leur statut, leur rôle, leur classification, leur nombre et répartition, Pierre Demarne nous donne les coractéristiques, le comportement et la philosophie des cadres américains et français en tentant quelque peu de les comparer

Toujours en poursuivant son étude comparative entre la France et les Etats-Unis, il expose les méthodes et programmes de perfectionnement technique, administratif et général existant dons l'entreprise et à l'université. Puis, il élabore ensuite un choix de programmes caractéristiques dans les domaines de la technique, de l'administration des affaires, de l'humanisme, de la culture supérieure et de la psychologie.

Une fois ces programmes établies dans l'entreprise, il est essentiel de pouvoir les évaluer et pour ce faire on doit élaborer une enquête globale d'évaluation des résultats. C'est ainsi qu'une telle enquête fut établie par les programmes universitaires américains pour mesurer la sensibilité des cadres à ces programmes.

L'auteur termine son ouvrage par une série de suggestions pouvant améliorer le perfectionnement des cadres en rapport ovec des points aussi divers que les auditoires, les animateurs, les programmes de communications, l'enseignement et le lieu d'enseignement.

Bref, ce volume constitue une contribution précieuse à l'amélioration de la connaissance sur un sujet de plus en plus développé à sovoir la formation professionelle. II servira certes à rehausser la qualité de l'utilisation des ressources humaines de l'entreprise.

\section{Ronald Pleou}

Plaidoyer pour la grande organisation, Léonard R. Sayles, Entreprise Moderne d'Edition, Paris, 1965, 141 pages.
Contrairement d̀ ce qu'en pensent certains, il est faux de prétendre que l'homme est "écrasé » par la grande entreprise et la grande administration. Malgré la complexité de cette dernière, I'individu subsiste et subsistera toujours avec tous ses attributs tels sa créativité, son initiative, son jugement. Personne d'ailleurs ne doutera de la valeur de ces qualités personnelles pour l'entreprise. Aussi ni lui ni elle n'ont à craindre de collaborer pour de plus grandes réalisations.

L'emploi abusif de l'initiative (exercée à l'encontre des projets de la collectivité) peut réduire l'efficacité d'une entreprise, mais se soumettre à trop de conformisme mène aux mêmes résultats. Aussi est-il nécessaire pour la société industrielle de trouver le juste milieu entre ces deux extrêmes et $d^{\prime}$ \& encourager au maximum l'initiative et la prise de risques calculés chez ses membres »

C'et pour nous renseigner davantage sur ces considérations que le professeur Sayles a composé cet ouvrage. Son but est d'étudier le travail des dirigeants lequel pose des problèmes au même titre que le trovail de production.

Il a divisé son travail en trois parties bien distinctes. Dans la première, il passe en revue la nature des grandes organisotions. Où se situe la liberté individuelle à l'intérieur de celle-ci? Quels sont les rapports entre la soustraitance et les relations employeurs-salariés? Quelles sont les sources insolites d'initiative et de créativité? Quels sont les problèmes de manipulation du personnel? Voilà les questions auxquelles l'auteur tente de donner de brèves réponses.

Dans une deuxième, il est traité de l'organisation comme facteur de changement chez l'être humain. Le rend-t-elle plus terre à terre, moins innovateur, moins désireux de s'engager dans l'action? Si oui, peut-on élaborer une théorie du conflit personnalitéorganisation? L'étude des motivations de I'homme à son emploi et des conséquences de l'évolution sociologique nous aidera à éclcircir ce point.

Si le conflit existe, il y a sûrement moyen de l'éviter. C'est pourquoi l'auteur se plait dans une troisième section à considérer les différentes façons de récompenser l'individu pour son tolent, à tenir compte de la santé physique et mentale des individus travaillant dans la grande entreprise, de la psychiatrie industrielle, et des bons et mauvcis usages des idées créatrices. II démentit également la prétention qu'une corrélation négative 
existe entre le degré de structuration de l'entreprise et le degré de liberté dont jouissent ses membres. Enfin, il s'intéresse à I'organisation en tant que structure contribuant d̀ la promotion, ou développement de l'individu et à sa contribution oux objectifs de la société $\gg$.

Bref, on peut conclure avec le professeur Sayles qu'on ne peut accepter l'affirmation de ceux qui prétendent que la vie dans les grandes organisations conduit à un conformisme excessif et à la dépendance.

Dans cet ouvrage concis et très bien structuré, tout est remis en question concernant les notions traditionnelles de a staff $»$ et a line $»$. On en arrive a la conclusion que si l'on s'y arrête et qu'on réfléchit le moindrement dे ce qui se passe dans les grandes organisations, on ne peut plus maintenant diviser les services de ces dernières en deux types bien délimités.

Une phrase du compositeur mentionne d'ailleurs que: «es conclusions réunies dans cet ouvrage, affirment que la grande organisation industrielle reconcilie l'initiative individuelle avec les nécessités de l'organisation, en donne une image très différente de celle habituellement présentée ou public .

\section{Ronald Pleau}

\section{Profit Sharing in Perspective, B.L. Metzger}

Profit Sharing Research Foundation, 1718

Sherman Avenue, Evanston, Illinois, 1964, 158 poges.

Cette étude sur la distribution des profits dans les petites et moyennes entreprises aux Etots-Unis est le résultat de deux ans de recherche sur les différentes pratiques qui y sont établies. Elle consiste en une enquête qui fut menée dans le but de déterminer l'étendue et l'incidence des programmes de distribution des profits. Cette enquête fut ensuite suivie d'une étude des caractéristiques, des objectifs, des résultats et déficiences des différents plans dont l'acceptation est de plus en plus accentuée.

Quelques-unes de ces découvertes sont assez surprenantes. Ainsi, par exemple, une compagnie sur cinq de celles ayant 50 employés ou plus possède son plan de partoge des profits, $70 \%$ de ces plans sont des programmes couvrant la majorité des employés réguliers et plus de 8 plans sur dix ont été évalués comme étant des succès.

On porte une attention particulière dans différents chapitres à d'autres découvertes de cette enquête, d̀ l'attitude changeante de ceux qui formulent la politique des syndicats et à l'étonnante accélération de la croissance des systèmes de partage des profits.

Comme le mentionne l'auteur, la distribution ou le partage des profits a été défini comme étant une méthode d'augmenter le rendement et diminuer les coûts par lo coopération humaine qui s'opère par la participation directe des employés aux résultats totaux de l'entreprise mesurés par les profits. II existe donc trois types principaux de plans concernant ce sujet lesquels sont décrits dans un premier chapitre.

Puis dans les suivants, c'est la description de la procédure suivie pour l'enquête préliminaire de même qu'une étude des types de programmes de partoge des profits que I'on rencontre le plus souvent dans l'entreprise américaine, de ses caractéristiques générales et de la participation des employés à ces plans.

Cette première partie est couronnée par un échantillon des plans i.e. un genre de tableau sommaire des différents plans étudiés dans les chapitres précédents.

Dans ce qu'on pourrait appelée une seconde partie, on analyse les résultats de cette enquête et ce sont les objectifs du plan, l'évaluation qu'en fait le management, les effets des programmes, l'attitude du syndicat vis-à-vis le partage des profits et enfin les conditions de succès de ces plans et programmes qui sont l'objet d'un examen plus approfondi. Un dernier chapitre vient compléter cette analyse avec une brève étude des autres genres de programmes de bénéfices.

De courts résumés à la fin de chaque chapitre sont présentés dans le but de fournir au lecteur une image globale ou permettre une révision rapide des buts du chapitre bien qu'ils soient loin de constituer des substituts à l'information beaucoup plus détaillée contenue dans ces derniers.

\section{Ronald Pleau}

Action Research for Management, by William Foote Whyte and Edith $L$. Hamilton, Richard D. Irwin, Inc., Homewood, Illinois, 1964, 282 pages.

Publié en fonction des gens intéressés aux relations humaines dans l'industrie, à la sociologie industrielle et à l'administration du personnel, ce volume est un compte-rendu d'un projet de recherche dan!: l'industrie des 